\title{
Lumen
}

Selected Proceedings from the Canadian Society for Eighteenth-Century Studies

\section{Français canadiens ou Canadiens? Construction et mutation d'une identité originale au XVIII ${ }^{\mathrm{e}}$ siècle}

\section{Réal Ouellet}

Volume 21, 2002

URI : https://id.erudit.org/iderudit/1012266ar

DOI : https://doi.org/10.7202/1012266ar

Aller au sommaire du numéro

Éditeur(s)

Canadian Society for Eighteenth-Century Studies / Société canadienne d'étude du dix-huitième siècle

ISSN

1209-3696 (imprimé)

1927-8284 (numérique)

Découvrir la revue

Citer cet article

Ouellet, R. (2002). Français canadiens ou Canadiens? Construction et mutation d'une identité originale au XVIII ${ }^{\mathrm{e}}$ siècle. Lumen, 21, 21-43.

https://doi.org/10.7202/1012266ar

Copyright (C Canadian Society for Eighteenth-Century Studies / Sociéte canadienne d'étude du dix-huitième siècle, 2002
Ce document est protégé par la loi sur le droit d'auteur. L'utilisation des services d'Érudit (y compris la reproduction) est assujettie à sa politique d'utilisation que vous pouvez consulter en ligne.

https://apropos.erudit.org/fr/usagers/politique-dutilisation/ 


\section{Français canadiens ou Canadiens? Construction et mutation d'une identité originale au XVIII siècle}

Pour bien comprendre comment naît et se développe une identité canadienne originale, il faut d'abord rappeler une évidence première touchant la colonisation et le peuplement de l'Amérique du Nord ${ }^{1}$. Dès le départ, les entreprises française et anglaise diffèrent grandement. La colonisation anglaise est d'abord le fait de petites communautés religieuses compactes qui s'installent dans les régions côtières atlantiques pour cultiver les terres ou faire le commerce. Ce faisant, elles entrent en conflit immédiat avec les groupes amérindiens qui y sont installés. Quand, à la même époque, les Français occupent et cultivent les terres de la vallée du Saint-Laurent, ils ne provoquent le départ d'aucun groupe amérindien sédentaire, puisque aucun n'y est installé. D'autre part, le commerce des fourrures, qui demeure jusqu'à la fin du Régime français une activité essentielle à la survie de la colonie, incite à l'expansion, car ce sont les Français établis dans la vallée du Saint-Laurent qui acheminent les fourrures jusqu'aux comptoirs de traite, en allant les chercher directement chez les nations amérindiennes de l'intérieur du continent. Très tôt s'établit donc une tradition de nomadisme qui touche non seulement les coureurs de bois proprement dits, mais aussi bon nombre de colons qui participent à la traite pendant leur jeunesse, ou encore deviennent voyageurs durant les mois d'hiver. En 1685, le gouverneur Denonville évalue à 800 , sur une population de 10000 à 12000 habitants, le nombre des coureurs de bois.

Le tiraillement entre deux manières d'exploiter le territoire - l'agriculture et le commerce des fourrures - a vite marqué la population

1 Je dois beaucoup aux collègues qui m'ont fourni de la documentation (surtout Martin Fournier et Simon Langlois) et à Denys Delâge dont les suggestions ont été précieuses. Je les remercie vivement de leur générosité. 
d'origine française, partagée entre la vallée laurentienne, à dominante sédentaire, et les pays d'En-Haut (la région des Grands Lacs), où les voyageurs nomades se mêlent aux Amérindiens avec lesquels ils commencent à se métisser. Ce ne sont pas seulement des alliances formelles et durables qui ont été forgées avec les Amérindiens, mais des manières d'être, de vivre et même de penser. Éparpillés et peu nombreux sur un espace qui n'est qu'une "vaste forêt» trouée de lacs et de rivières mais privée de routes, entourés de groupes amérindiens beaucoup plus nombreux qu'eux, les premiers colons laurentiens apprirent à chasser le gros et le petit gibier, à pêcher sous la glace, à se déplacer en raquettes et en canot d'écorce de bouleau, à se nourrir de blé d'Inde, de fruits sauvages et de chair d'animaux boucanée. Ils prirent aussi l'habitude de fumer, de chiquer et de priser le tabac qu'ils cultivaient eux-mêmes. Dans leurs conflits armés avec les Iroquois et les Anglais, ils utilisèrent vite la stratégie de la guerre à l'indienne, faite d'embuscades, d'attaques surprises en petits groupes et de retraits rapides. Enfin, peut-être est-ce à l'époque de la Nouvelle-France que les Sauvages, et non pas les cigognes, commencèrent à apporter les bébés aux mères canadiennes: «Les Sauvages sont encore passées chez Mme Bouchard», disait une voisine à ma mère quand $j$ 'étais enfant.

Les autorités coloniales elles-mêmes, dans un premier temps, encouragèrent le métissage systématique entre Amérindiens et colons français, d'une part à cause du grave déséquilibre démographique entre hommes et femmes $d$ 'origine française dans la colonie, $d$ 'autre part parce qu'elles croyaient ainsi «civiliser» plus rapidement les Sauvages, en faire autant de bons sujets français. Si les administrateurs renoncèrent vite à cette entreprise utopique de fusion, qui confondait nomades et sédentaires, plusieurs colons partagèrent jusqu'à un certain point la mentalité et les manières de faire amérindiennes.

Enfin, on peut juger comme on voudra l'entreprise des missionnaires français: il reste que ces hommes, par leur labeur quotidien et continu de deux siècles, ont acquis une connaissance profonde, existentielle, des nations amérindiennes. En ce sens, les jésuites Brébeuf et Le Jeune sont des écrivains importants et aussi de grands ethnologues, le premier chez les Hurons, le second chez les Montagnais². Lafitau, un autre

2 Brébeuf, Relation de ce qui s'est passé dans le pays des Hurons en l'année 1636, chapitre 6, dans The Jesuit Relations and Allied Documents, édit. R. G. Thwaites, Cleveland, The Burrows Bros Co, 1896-1901, vol. 10, p. 210-249 (réimprimé en fac-similé par Pageant Books, New York, 1959); Paul Le Jeune, Relation de ce qui s'est passé en la mission des peres de la Compagnie de Jesus aux païs de la Nouvelle France, depuis l'esté 1657 jusques à 
jésuite, missionnaire chez les Iroquois du Saut-Saint-Louis, publia en 1724 Mours des sauvages américains, texte fondateur de l'anthropologie comparée $^{3}$. Malgré leurs certitudes religieuses, ces missionnaires-anthropologues finirent par adopter une position relativisante parce qu'ils se sont retrouvés en position d'interculturalité étroite avec la population amérindienne. Les Anglais connurent aussi ce type de rapport interculturel dans la baie d'Hudson, mais sur une échelle beaucoup plus réduite. Ces mêmes Anglais prendront vivement conscience de leur méconnaissance du territoire et de leur distance des Sauvages quand, après la Conquête, ils auront recours aux Canadiens d'ascendance française pour traiter avec les Amérindiens de Détroit et de la région des Grands Lacs.

Dans ces conditions, faut-il se surprendre que très tôt apparaisse une distanciation culturelle progressive des Canadiens à l'égard de leur société d'origine dont ils se sentent différents par tout un système de valeurs et de contraintes d'ordre politique, juridique et religieux? Le sentiment $d$ 'appartenance au nouveau territoire, qui se manifeste très tôt dans les comportements (la Communauté des Habitants, par exemple), trouvera son écho dans les rapports des gouverneurs et dans des témoignages comme celui de l'officier d'infanterie Duplessy Faber, pour qui les Canadiens et les Français sont de «deux nations différentes ${ }^{4}$ ». C'est l'émergence de cette construction graduelle d'une identité originale que je voudrais analyser dans les correspondances officielles et privées, les relations de voyages et autres écrits sur la Nouvelle-France publiés sous le Régime français, puis dans divers ensembles textuels de la seconde moitié du XVIII ${ }^{\mathrm{e}}$ siècle $^{5}$.

l'année 1658, chapitre 7, dans The Jesuit Relations and Allied Documents, vol. 44, p. 276-309.

3 Joseph-François Lafitau, Maurs des sauvages américains comparées aux maurs de premiers temps, Paris, Saugrain l'ainé et Charles Estienne Hochereau, 4 vol., 1724.

4 Lettre à Vauban, 16 septembre 1698, dans la Correspondance de Vauban relative au Canada, édit. L. Dechêne, Québec, ministère des Affaires culturelles, 1968, p. 16.

5 Sur la construction d'une identité canadienne à l'époque de la Nouvelle-France en rapport avec l'indianité, voir Denys Delâge, «L'influence des Amérindiens sur les Canadiens et les Français au temps de la Nouvelle-France», Lekton, automne 1992, p. 103-191; «Essai sur les origines de la canadianité», séminaire de la CEFAN, 15 juillet 1997, 25 p., inédit. Pour une analyse globale, des origines à nos jours, voir Simon Langlois, "Canadian Identity: a Francophone Perspective», dans Encyclopædia of Canada's People, Toronto, University of Toronto Press, 1999, p. 323-329; Réal Ouellet, «Identité québécoise, permanence et évolution», dans A. Beaulieu et M. Tremblay (édit.), les Espaces de l'identité, Québec, Presses de l'Université Laval, 1997, p. 62-98. Il 


\section{Le Régime français}

La période cruciale de consolidation de cette identité canadienne se situe vers $1680-1690^{6}$. De son arrivée au Canada en 1685 jusqu'à son départ en 1689 , le gouverneur Denonville, estimant que la colonie s'est affaiblie en s'étendant vers l'ouest par le commerce des fourrures, veut la recentrer sur la vallée du Saint-Laurent avec l'agriculture et la pêche trop négligées. Plus qu'un simple éparpillement, le commerce des fourrures entraîne une déperdition des forces françaises, parce que, au lieu de civiliser les Sauvages, "de les accoûtumer à nos $\operatorname{lois}^{7}$ ", les coureurs des bois s'ensauvagent eux-mêmes. Quand il rentrera en France dans les derniers jours de 1689, il insistera une dernière fois sur l'effet social désintégrateur de la traite des fourrures qui développe chez les coureurs des bois un esprit d'indépendance et de liberté incompatible avec le type de société que veulent maintenir les élites de l'époque:

le grand nombre de coureurs des bois a fait un notable préjudice à la colonie en corrompant l'esprit, le corps et les mours des habitants qui, s'entretenant dans l'esprit libertin et indépendant et fainéant, empêche qu'ils ne se marient, car l'air de noble qu'ils prennent à leur retour par leurs ajustements et leurs débauches au cabaret, dépensant ainsi tout leur profit en très peu de temps, fait que, méprisant les paysans, ils tiennent au-dessous d'eux d'épouser leurs filles, bien qu'eux-mêmes soient paysans comme eux, et outre cela ne se veulent plus abaisser à cultiver la terre et ne veulent plus entendre qu'à retourner dans les bois continuer le même métier, ce qui donne lieu à quantité de débauches que plusieurs font avec les Sauvagesses, qui attirent beaucoup de maux par les déplaisirs que les Sauvages ont qu'on débauche leurs femmes et filles et par le tort que cela fait à la religion, les Sauvages voyant que les Français ne pratiquent rien de ce que les missionnaires disent de la loi de l'Évangile ${ }^{8}$.

Cette condamnation du nomadisme insiste surtout sur la corruption de l'esprit par la débauche: en nomadisant, l'habitant canadien devient

faudrait aussi citer les livres importants de F. Dumont, G. Bouchard, J. Létourneau et quantité d'autres.

6 C'est ce qui se dégage aussi du livre de Gervais Carpin, Histoire d'un mot. L'ethnonyme «Canadien» de 1535 à 1691, Sillery (Québec), Septentrion, 1995.

7 Lettre de Denonville à Seignelay, 13 novembre 1685, Archives des colonies, C11A, 7, f. 90vo.

8 Mémoire de Denonville à Seignelay, janvier 1690, Archives des colonies, C11A, vol. 11, f. 187 vo-188ro. 
«indépendant», «libertin» et «fainéant». Les trois termes sont importants. Alors que libertin désigne surtout la conduite déviante, $l^{\prime}$ «indépendance d'esprit» suggère une attitude incompatible avec le système monarchique français fondé sur la hiérarchie sociale et l'obéissance absolue à l'autorité. La «fainéantise», enfin, s'oppose ici au travail assidu et régulier du sédentaire et risque d'appauvrir et de paralyser toute la collectivité. Cette condamnation morale atteint, on le voit, le politique et le social. Sur le plan politico-économique, le nomadisme freine l'action missionnaire qui a partie liée avec le développement colonial et met en péril le fragile équilibre commerce/agriculture de la colonie. Sur le plan social, le nomadisme perturbe l'ordre établi, puisque, d'une part, le nomade veut sortir de son rang quand il prend «l'air de noble» et refuse d'épouser une fille d'habitant et que, d'autre part, il gaspille dans la débauche à la fois son patrimoine, au lieu de le consolider, et sa précieuse semence, nécessaire pour assurer la croissance démographique.

Si le nomadisme de plusieurs Canadiens représente un danger considérable pour la cohésion sociale et le développement de la colonie, il a aussi forgé des miliciens capables de résister au climat rigoureux et de compenser l'infériorité numérique dans laquelle la France se trouve face aux Anglais et aux Iroquois. Régulièrement, dans leur correspondance avec le Ministre, gouverneurs et intendants vantent les qualités militaires des Canadiens qui aiment se battre à la sauvage, c'est-à-dire fondre sur l'ennemi par surprise puis se retirer rapidement pour se mettre à couvert. C'est bien ce qui se passera lors des trois expéditions punitives lancés par Frontenac, en 1690, contre les villages frontaliers de Corlaer (Schenectady, N.Y.), Salmon Falls (côte du Maine) et fort Loyal (Portland, Maine), où des détachements constitués de Canadiens et d'Indiens jetèrent la terreur quand ils brûlèrent les maisons et les fermes, massacrèrent ceux qui tentèrent de résister et ramenèrent plusieurs prisonniers. De là une réputation de cruauté, aussi bien chez les Anglais de la Nouvelle-Angleterre que chez les Français ${ }^{9}$. De là aussi une réputation de tête brûlée, hostile à toute discipline, comme le verra bien le chevalier

9 Le 20 janvier 1699, dans une lettre à Vauban, Maurepas parlera de leur «férocité insurmontable contractée par la fréquentation continuelle qu'ils ont avec les Sauvages» (la Correspondance de Vauban, p. 32). Outrée par la cruauté des opérations, la General Court, qui gouvernait le Massachusetts, discuta à plusieurs reprises d'une attaque d'envergure contre la colonie française, pour stopper la «bloody war» que menaient les "Français» et leurs alliés amérindiens (The Glorious Revolution in Massachusetts. Selected Documents, 1689-1692, édit. R.E. Moody et R.C. Simmons, Boston, The Colonial Society of Massachusetts, 1988, p. 236). 
de Troyes lors de son expédition de 1686 contre les Anglais de la baie d'Hudson. Si l'opération fut un succès, grâce à «la fougue de nos Canadiens [...] faisant de grands cris à la manière des sauvages ${ }^{10}$ ", le chevalier de Troyes en a gardé la conviction que «le naturel» des Canadiens «ne s'accorde guère avec la subordination", comme le révèle une anecdote qu'il raconte dans son Journal: voulant "attacher un Canadien à un arbre pour le punir de quelque sottise qu'il avait dite», "quelques mutins voulurent à ce sujet exciter une sédition» qu'il eut grand-peine à calmer, avec l'aide du missionnaire Silvy (Journal, p. 31).

Ce portrait du Canadien, qui court en filigrane dans la correspondance officielle des années 1680-1700, se retrouvera pendant le demisiècle suivant, à quelques variantes près, sous la plume d'administrateurs, de romanciers, de voyageurs, d'officiers militaires ou de missionnaires comme Bacqueville de la Potherie, Charlevoix et Kalm. De ces témoignages bien connus, je ne retiendrai que ce qui touche l'homogénéisation sociale et une certaine forme de civilité. Dans l'importante lettre 12 du tome 1 de son Histoire de l'Amérique septentrionale, publiée en $1722^{11}$, Bacqueville de la Potherie laisse un portrait très vif des hommes et des femmes du Canada qu'il connut lors de son séjour à titre de commissaire puis de contrôleur de la Marine, entre 1698 et 1701. Après avoir parlé de leur fierté, de leur indépendance et finesse d'esprit, de leur habileté manuelle, de leur goût pour la guerre et la dépense, il trace un portrait de la Canadienne qui sera souvent repris par la suite et qu'on trouvera aussi bien chez Saugrain ${ }^{12}$ que chez Charlevoix ${ }^{13}$ : "ce sexe y est aussi poli qu'en aucun lieu du royaume. La marchande tient de la femme de qualité, et celle d'officier imite en tout le bon goût que l'on trouve en France» (Histoire, p. 368). Ce qu'affirme Bacqueville, c'est une civilité, un bon goût et des manières dignes de la France raffinée, mais aussi l'appartenance à une collectivité où la hiérarchie sociale tend à s'effacer, tout

10 Chevalier de Troyes, Journal de l'expédition du chevalier de Troyes à la baie d'Hudson, en 1686, édit. I. Caron, Beauceville, La Compagnie de «L'éclaireur», 1918, p. 67.

11 Bacqueville de la Potherie, Histoire de l'Amérique septentrionale, Paris, Jean-Luc Nion et François Didot, 1722.

12 Saugrain, Dictionnaire universel de la France ancienne et moderne, et de la Nouvelle-France, Paris, Saugrain Père et la Veuve J. Saugrain, Pierre Prault, 1726.

13 Charlevoix, Histoire et description générale de la Nouvelle-France, Paris, Nyon Fils, 3 vol., 1744; voir particulièrement le vol. 3, Journal d'un voyage fait par ordre du roi dans l'Amérique septentrionale, édit. P. Berthiaume, Montréal, Presses de l'Université de Montréal, coll. «Bibliothèque du Nouveau Monde», 1994, p. 234-235, 245-257, 393, 402-405. 
particulièrement chez les femmes de Montréal, plus «libres» et "franches» que celles de Québec, qui manifestent «beaucoup sur la réserve» (ibid., p. 368). Cette homogénéisation, si perceptible chez les femmes, se reflète aussi dans la langue française qu'on y "parle ici parfaitement bien, sans mauvais accent» et qui est «un mélange de presque toutes les provinces de France» (ibid., p. 279).

Des observations similaires se retrouvent en 1749 chez le botaniste suédois Pehr Kalm, selon lequel «il existe ici un brin de liberté innocente et que les femmes sont dépourvues de toute timidité paysanne. La plupart d'entre elles sont, au contraire, bien élevées et polies au suprême degré $^{14}$.» Tout comme Charlevoix et Bacqueville, Kalm reprend la comparaison en mettant l'accent sur une différence de sociabilité: les femmes de Québec «sont assez proches, en matière de savoir-vivre, des femmes venues de la vieille France; mais on trouve chez les femmes de Montréal une sorte de fierté sauvage» (Voyage, p. 315). Cette métaphore de la sauvagerie se trouvait un peu plus tôt exprimée de manière plus vive, directe, lorsque Kalm reprenait les propos de «Français nés en France et venus s'installer ici», selon lesquels les Montréalaises, par opposition aux Québécoises, manqueraient «de la bonne éducation et de la politesse française d'origine», parce qu'elles seraient "poussées par un certain orgueil et comme contaminées par l'esprit imaginatif des Sauvages d'Amérique» (ibid., p. 198-199).

Kalm était bien placé pour porter un jugement sur la société canadienne. Venu en Amérique du Nord pour y étudier la flore, il passa quelques mois en Nouvelle-France (en 1749), où il fréquenta, non seulement l'élite, mais aussi et plus volontiers l'habitant. Dans son Journal de voyage, publié d'abord en suédois (1753-1761), on sent une curiosité bienveillante qui le porte à tout voir de ses propres yeux. Bien reçu par le gouverneur général de Québec et par l'élite de la colonie, il en vante "l'extrême politesse», toute différente de celle qu'il a trouvée dans les "provinces anglaises»: entre Anglais et Canadiens, «il y a toute la différence qui sépare le ciel de la terre, le blanc du noir, et cela en tous les domaines» (ibid., p. 182). Mais c'est chez l'«habitant» et chez les petites gens qu'il se plaît davantage. Il note au passage leur goût exagéré du paraître dans l'habillement, la liberté de parole des femmes, mais

14 Pehr Kalm, Voyage de Pehr Kalm au Canada en 1749, édit. J. Rousseau, G. de Béthune et P. Morrisset, Montréal, P. Tisseyre, 1977, p. 183. Sur Kalm et les "Canadiens», voir l'étude de Michel Bideaux, «L'émergence d'un ethnotype créole: le Canadien du XVIII ${ }^{\mathrm{e}}$ siècle», dans The Paths of Multiculturalism. Travels Writings and Postcolonisation, édit. M. Seixo et autres, Lisbonne, Cosmos, 2000, p. 263-274. 
surtout leur gaîté et leur optimisme: «je n'ai pas rencontré de gens qui soient aussi drôles qu'eux, toujours gais et de bonne humeur, profondément courageux et qui tiennent que rien n'est impossible à surmonter» (ibid.,p. 413). Il insiste avant tout surl'hospitalité et les manières courtoises des habitants, "qu'on ne trouve jamais chez les gens du commun de la campagne française», au témoignage de "Français natifs de Paris»: «Les gens du commun au Canada sont plus civilisés et plus ingénieux qu'en n'importe quel autre endroit du monde où je me suis rendu» (ibid., p. 546). Faut-il attribuer cette courtoisie et ce savoir-vivre à leur aisance économique (ibid., p. 415)? Pour ma part, je serais porté à y voir un indice de plus que les différences entre les classes sociales avaient tendance à s'atténuer chez les Canadiens à la veille de la conquête anglaise. Dans un autre passage sur la politesse des paysans, Kalm mentionne que ceux-ci empruntent à la fois à la France civilisée et au monde sauvage: $d$ 'une part, ils «possèdent d'assez jolis» «ustensiles de ménages» et ils «se servent de fourchettes pour manger» (ibid., p. 415); d'autre part,

les Français canadiens de condition modeste ont assez souvent adopté la mode et les coutumes des Sauvages d'Amérique, par exemple en ce qui concerne les pipes, les chaussures, les bandes molletières, les ceintures, la façon de courir en forêt, les méthodes de guerre, [...] les bateaux en écorce [...] (ibid., p. 414).

Quand le commissaire de la marine Bacqueville de la Potherie, l'historien jésuite Charlevoix et le botaniste Pehr Kalm établissent un rapport de ressemblance, voire d'interculturalité entre Canadiens et Sauvages, on ne peut le rejeter d'emblée, car on le trouve aussi exprimé à la même époque par une Canadienne, la Montréalaise Élisabeth Bégon émigrée en France: «ll paraît, écrit-elle le 20 janvier 1750, que je ne sais point la façon dont on doit vivre et que je ne suis qu'une Iroquoise ${ }^{15}$.» Elle, qui a été en Canada la femme du gouverneur de Trois-Rivières, qui a connu les plaisirs de la vie mondaine, qui a frayé avec les personnages importants de la colonie, se retrouve à La Rochelle et à Rochefort marginalisée, rejetée par la noblesse provinciale française. Tout la gêne et tout l'irrite: le froid dans des maisons qu'on n'arrive pas à chauffer; les «misérables chaises à porteurs» et les "misérables rues» où l'on ne peut guère

15 Élisabeth Bégon, Lettres au cher fils, édit. N. Deschamps, Montréal, Hurtubise HMH, 1972, p. 205. 
marcher (20 février 1750, p. 210); même la difficulté à trouver une «bonne plume» pour écrire (14 mai 1750 , p. 227)!

Avant de passer au Régime anglais, $j$ 'aurais voulu citer deux autres témoignages, fort importants dans la mesure où ils se situent juste au moment de la conquête anglaise du Canada: ceux des officiers militaires français Bougainville et d'Aleyrac. Je ne citerai que quelques phrases du premier qui montrent bien que, pendant les dernières années de la guerre de Sept Ans, l'animosité deviendra si grande, entre Français et Canadiens, qu'elle perturbera le déroulement des opérations militaires.

D'abord aide de camp du général Montcalm, Bougainville arrive au Canada à 27 ans. Il vient tout juste d'entrer à la prestigieuse Royal Society de Londres grâce à un ouvrage de vulgarisation mathématique intitulé Traité de calcul intégral et publié deux ans plus tôt ${ }^{16}$. Imbu d'idées philosophiques, il a fréquenté le salon de Mme du Deffand où il a rencontré D'Alembert, Fontenelle, Helvétius, Diderot, Hume. Et c'est bien en philosophe qu'il prétend étudier la réalité sauvage nord-américaine, «un philosophe qui cherche à étudier l'homme dans ceux surtout qui sont les plus voisins de la première nature ${ }^{17}{ }$. Mais dès son arrivée, saisi par la tourmente guerrière, il prend parti dans le conflit qui oppose militaires français et canadiens. A l'instar de bien d'autres administrateurs ou voyageurs métropolitains avant lui, Bougainville voit les Canadiens non pas comme des frères ou des cousins, mais comme les proches parents des Amérindiens. Sous sa plume, les termes Canadiens et Sauvages voisinent souvent comme les hommes voisinaient aussi sur le terrain. De toute évidence, il a d'abord vu le Canadien à travers une image de l'Amérindien. Et l'Amérindien qu'il a découvert en Amérique n'a rien à voir avec le bon Sauvage de Rousseau ni le doux primitif qui l'accueillera à Tahiti en 1768 , lors de son célèbre voyage autour du monde. C'est d'abord un guerrier sanguinaire et anthropophage (Écrits, p. 228, 318, $399,405)$, un être indiscipliné et pillard, un «monstre» (p. 400, 408, 416) sans honneur ni courage, ni même stratégie militaire, guidé par le seul instinct de vengeance (p. 161-162). Au témoignage des Anglais, les Canadiens sont si cruels qu'en cas de victoire sur les forces françaises «il y aura deux capitulations, une pour les troupes françaises et l'autre pour les Canadiens» (septembre 1757, p. 420). Aux yeux du jeune officier

16 Bougainville, Traité du calcul intégral pour servir de suite à l'analyse des infiniments petits de M. le marquis de l'Hôpital, Paris, H.-L. Guérin et L.-Delatour, 2 vol., 1754-1756.

17 Bougainville, «Journal de l'expédition d'Amérique commencée en l'année 1756, le 15 mars", dans Écrits sur le Canada: Mémoires - Journal - Lettres, édit. R. Lamontagne, Sillery (Québec), Éditions du Pélican, 1993, p. 122. 
français, il existe une bonne et une sale guerre: la bonne, c'est une «belle et glorieuse campagne» (ibid., p. 420), qui apporte «un succès sans tache» (20 février 1758, p. 424); la sale guerre détruit tout sur son passage, ne fait pas de quartier à l'ennemi. Sur ce plan, Français et Anglais appartiennent à la même culture européenne, tandis que les Canadiens et «leurs frères les Sauvages» (p. 422), sont relégués dans la barbarie. On comprend alors que Bougainville insiste à ce point sur certaines scènes où l'on voit des Français se chamailler avec des Canadiens et des Sauvages pour libérer des prisonniers anglais, au risque même de leur vie (p. 419), ou encore rivaliser de politesse ensemble, quand l'envoyé anglais apporte à Bougainville «un panier de bière de Bristol de la part du cap ${ }^{\text {ne }}$ Abercromby» et que l'officier français lui donne du «vin de Pacaret» (p. 343). On comprend aussi l'embarras de Bougainville, sa «honte» (p. 422), nous dit-il à plusieurs reprises, d'avoir «pour compagnons d'armes des monstres capables de nous déshonorer», des alliés dont l'«infamie souille notre gloire» (p. 416).

\section{La conquête anglaise}

Que devient l'identité canadienne quand la France cède sa colonie à l'Angleterre par le traité de Paris en 1763? Arrêtons-nous un moment sur le contexte historique. Une certaine tradition historiographique, souvent en réaction contre les historiens cléricaux du $\mathrm{XIX}^{\mathrm{e}}$ siècle et contre la position des Frégault, Brunet et Séguin, a minimisé l'importance de la conquête anglaise, comme si les «nouveaux sujets» étaient passés d'un régime à l'autre sans douleur ni traumatisme important. Il faut ici rappeler quelques faits. La conquête de 1759-1760 met un terme à la longue guerre de Sept Ans, désastreuse à tous points de vue. Cette guerre a fait près de 10000 morts, soit 13 ou $14 \%$ de la population d'origine française. Elle a ravagé les villages côtiers du Saint-Laurent à l'est de Québec jusqu'à Rivière-Ouelle sur la rive sud, et jusqu'à Baie-Saint-Paul sur la rive nord; elle a détruit une bonne partie de la ville de Québec. Imaginons seulement l'hiver 1759-1760. Seule la région de Québec est aux mains des Britanniques: une population de 8000 habitants doit loger 6000 soldats anglais qui sont leurs ennemis héréditaires; elle est coincée entre un conquérant sur place et des compatriotes, installés vingt-cinq milles à l'ouest, qui la menacent tous deux de représailles; elle souffre de la misère par suite des destructions et des maigres récoltes avec lesquelles il faut aussi nourrir le conquérant. Sur le plan psychologique, enfin, pensons à l'humiliation des habitants (que les Anglais, aussi bien que les Français, disent fiers, voire arrogants) qui doivent rendre leurs fusils aux autorités britanniques. Sans aucun doute, ils pensaient aussi 
aux 13000 Acadiens que les Anglais venaient d'arracher à leurs terres pour les disperser brutalement aux quatre coins de leurs colonies. On en a dénombré 1015 réfugiés aux environs de Québec, mais qui ne purent obtenir les droits accordés aux «Canadiens». On imagine facilement chez ceux-ci une sorte d'hébétude, d'attente angoissée de ce qui adviendra d'un territoire régi par la loi martiale.

Ajoutons enfin le rétrécissement du territoire sur tous les fronts. Après la conquête anglaise, le Canada, qui s'étendait auparavant de l'Atlantique aux Grands Lacs et comportait aussi un large territoire à l'est du Mississippi, est amputé de ses parties nord-est, est, ouest et centre-ouestsud d'où provenaient la plus grande partie des fourrures de la NouvelleFrance: il se réduit à une bande qui, correspondant à la propriété seigneuriale, longe les deux rives du Saint-Laurent, de la pointe est du lac Ontario (Kingston) jusqu'à la limite est de la péninsule gaspésienne. Mais cette quinzième colonie anglaise, appelée Province of Quebec, diffère des autres considérablement. On y trouve 80000 descendants de français catholiques, regroupés en paroisses, dont la majorité est propriétaire des terres qu'elle cultive, contre quelques centaines de protestants anglophones, soldats ou marchands. L'éducation, dispensée au secondaire par le Collège des jésuites à Québec et au primaire par diverses écoles, souffrira grandement de la Conquête, puisque, par exemple, le célèbre Collège des jésuites, accaparé par l'armée anglaise dès l'automne 1759, servira de caserne pour loger les soldats à partir de 1765 .

La Proclamation royale du 7 octobre 1763 , tout comme les instructions données au gouverneur lors de son entrée en fonction, entendent faire $\mathrm{du}$ Canada une colonie anglaise où les «nouveaux sujets» (les Canadiens), francophones et catholiques, passeront graduellement au protestantisme, aux lois et coutumes anglaises. D'une part, pour occuper une fonction officielle, ils devront abjurer le catholicisme en prêtant le Serment du test, institué un siècle plus tôt en Angleterre; d'autre part, la création d'une Chambre d'assemblée, formée exclusivement de Britanniques, exclura du gouvernement les Canadiens; enfin, l'immigration massive de sujets britanniques noiera la population d'origine française.

Cette vision londonienne de la réalité canadienne ne sera pas celle des premiers administrateurs sur place. Ainsi, Murray, se rendant compte qu'il est impossible de déporter et de disperser dans les autres colonies ou même d'assimiler rapidement une population aussi nombreuse, décide de se l'attacher en lui donnant un certain nombre d'avantages et de garanties. Dès son arrivée au pouvoir, il s'entoure de quelques propriétaires fonciers canadiens et de quelques administrateurs anglais, qui forment ce qu'on a appelé paradoxalement le French Party, en face duquel se dressera un groupe antagoniste formé surtout de marchands, l'English Party, qui voudra faire respecter scrupuleusement les disposi- 
tions de la Proclamation royale. L'administration anglaise sera d'autant plus poussée à s'attacher les «nouveaux sujets» que bientôt s'agiteront les «treize colonies» anglaises du sud, auxquelles pourraient s'allier les anciens colons français. Mais, ce faisant, elle mécontente les colons et marchands britanniques qui ne peuvent comprendre qu'on ménage à ce point des vaincus, "papistes" de surcroît. Ce mécontentement s'exprimera bien davantage encore avec l'arrivée de 30000 loyalistes après la victoire des insurgés américains en 1683. 6000 de ces quelque 30000 loyalistes s'établiront dans la Province of Quebec, où, formant 15\% de la population, ils changeront l'équilibre démographique et provoqueront une pression considérable sur les autorités britanniques.

Ce comportement des Britanniques, qu'on a souvent qualifié d'amical, tient selon moi à deux facteurs principaux. Il faut y voir une sorte de fair play des gouvernants britanniques, imbus de principes de justice hérités des Lumières françaises et anglaises, et qu'on voit souvent exprimée dans la correspondance officielle, sous la plume du solliciteur général Wedderburn, en 1772, par exemple (DRHCC, 1759-1791, p. $403^{18}$ ). Il révèle aussi une intelligence très vive de la situation canadienne, contrairement aux marchands qui veulent s'enrichir vite. Sans doute, comme le suppose Philip Lawson ${ }^{19}$, les gouvernants ne veulent-ils pas répéter la coûteuse expérience irlandaise. Ne soyons donc pas naïs ni angéliques. Aux yeux des autorités britanniques, les Canadiens se montrent gentils et courtois, ils ont été de bons soldats, certes. Mais ils sont ignorants et imprévisibles. Comme ils sont soumis à leurs seigneurs et à leur clergé appauvris par la Conquête, attachons-nous leurs seigneurs et leur clergé par des faveurs monnayées en argent sonnant ou en honneurs (Carleton à Shelburne, 20 janvier 1768, DRHCC, 1759-1791, p. 269). Bien plus, appliquons ici l'indirect rule qui a si bien fonctionné ailleurs, en associant partiellement les élites des vaincus à l'administration de la colonie. Il est symptomatique, par exemple, que Murray, entre Briand et Montgolfier, ait choisi le premier pour devenir évêque de Québec, parce qu'il pouvait, selon l'expression populaire, le faire manger dans sa main, tandis que Montgolfier appartenait à l'ordre des sulpiciens qui possédait des propriétés foncières immenses. $\mathrm{D}^{\prime}$ ailleurs, qui serait porté à teinter de couleurs trop roses le portrait du général-gouverneur se rappellera

18 J'abrège ainsi le titre suivant: A. Shortt et A. G. Doughty, Documents relatifs à l'histoire constitutionnelle du Canada, 1759-1791, Ottawa, Archives publiques du Canada, 1921.

19 The Imperial Challenge: Quebec and Britain in the Age of the American Revolution, Montréal et Kingston, McGill-Queen's University Press, 1989, p. 47. 
son premier hiver à Québec, quand il menace de représailles terribles les habitants tentés d'aider les troupes de Lévis qui marchent sur la ville: "Leurs familles, leurs maisons seront en proie aux soldats furieux ..." Dans une autre proclamation, il parle $\mathrm{d}^{\prime}$ «une vengeance sanglante». Et ce ne sont pas que des mots, puisqu'il écrit lui-même: «Vous avez sans doute entendu dire que j'ai pendu un capitaine de milice, que je détiens à bord d'un navire de guerre un prêtre et quelques jésuites qui doivent être envoyés en Grande-Bretagne.» Le 26 février 1760, il envoie le major Elliot «avec un détachement de 300 hommes [...] incendier une vingtaine d'habitations depuis la rivière des Etchemins jusqu'à la rivière Chaudière ${ }^{20}$ ». Le gros bout du bâton, on le voit, n'est jamais très loin de la carotte.

Ce portrait implicite des Canadiens dans les rapports des trois gouverneurs généraux qui assurent le gouvernement militaire jusqu'en 1763 ne correspond guère à ce que pensent la majorité des Britanniques qui habitent la nouvelle colonie, comme le suggère la pétition envoyée au roi par les marchands de Québec en 1764: ils se disent persécutés par le gouverneur Murray et se plaignent de la «partialité flagrante qui le pousse à susciter des factions, à prendre des mesures propres à entretenir la séparation entre les anciens et les nouveaux sujets de Sa Majesté et à encourager ceux-ci à demander des juges de leur langue» (DRHCC, 1759-1791, p. 204). On trouvera un témoignage encore plus éloquent dans le roman épistolaire de Frances Brooke, rédigé à Québec en 17661767 et publié à Londres en 1769: The History of Emily Montague ${ }^{21}$. Le portrait global des Canadiens n'y est guère nuancé. S'ils se révèlent hospitaliers et courtois, ils sont surtout ignorants, paresseux, sales, stupides, cruels et superstitieux (voir notamment les lettres des 9 juillet et 10 septembre).

Mais que disent les Canadiens sur eux-mêmes? Ont-ils conscience d'une identité collective? Aucun document privé ne permet de s'en faire une idée précise. On peut seulement le présumer en lisant la maigre correspondance officielle qu'ils adressent à Londres. Entre 1764 et 1774, dans les quelques pétitions qu'ils envoient aux autorités anglaises pour réclamer la "conservation des anciennes lois» et coutumes françaises, et leur "participation aux emplois civils et militaires», ils n'en appellent pas seulement à la «bonté royale» ou à la «tendresse paternelle» du monarque: ils parlent d'un «acte de justice», puis, quelques années plus tard, 
ils invoquent le «droit naturel» (DRHCC, 1759-1791, p. 196-197; p. 399400; p. 490-494; p. 749-751). Cet appel renouvelé au paternalisme des administrateurs coloniaux s'inscrit, certes, dans une tradition conservatrice qui cherche à établir une alliance directe avec le monarque; elle marque surtout une prise de conscience et un gain politique prospectif, car les Canadiens ont intégré à leur mode de pensée l'idée de «droit» qui leur était inconnue, me semble-t-il, sous le Régime français.

L'Acte de Québec, voté par le parlement britannique en 1774, satisfait leurs demandes: il abolit le Serment du test, élargit le Conseil qui sera formé de vingt membres dont huit Canadiens, rétablit les lois civiles françaises et réunit à la Province de Québec le Labrador et la région des Grands Lacs jusque dans la vallée de l'Ohio. De toute évidence, l'Acte de Québec voulait satisfaire les Canadiens et les empêcher de se joindre aux rebelles de la Nouvelle-Angleterre dont l'agitation avait provoqué six mois plus tôt le fameux Boston Tea Party. Mais, contrairement aux attentes du gouverneur, quand les armées révolutionnaires américaines marchèrent sur le Canada, la masse des Canadiens refusa de s'enrôler et certains même se joignirent aux insurgés. Rien n'y fit: ni les exhortations de leurs seigneurs ni les menaces d'excommunication de leur évêque Briand qui leur demandait «seulement un coup de main pour repousser l'ennemi et empêcher l'invasion dont cette province est menacée ${ }^{22}{ }^{\prime}$.

Malgré l'empressement des seigneurs et du haut clergé, les habitants canadiens, comme s'en plaint amèrement Carleton dans sa lettre du 7 juin 1775 au secrétaire d'État Darmouth, refusent de se mobiliser: «bien que les gentilhommes aient manifesté beaucoup d'empressement, ils n'ont pu gagner le peuple»; «tout esprit de subordination est détruit et le peuple est empoisonné par l'hypocrisie et les mensonges». Il regrette même «d'avoir recommandé l'introduction de l'acte d'habeas corpus et des lois criminelles anglaises», parce qu'on en a "fait une arme contre l'État» (DRHCC, 1759-1791, p. 651-652). Mais, dans son désenchantement, Carleton oublie, me semble-t-il, une donnée psychologique fondamentale: comment les Canadiens ne seraient-ils pas humiliés, non seulement d'avoir été vaincus, mais aussi de devoir chanter, pour survivre, les louanges du vainqueur? Personne n'était dupe de cette rhétorique où vainqueurs et vaincus d'hier rivalisent de formules de «tendresse» mutuelle, d'affection "paternelle», d' «excès de bonté», où la fausse soumission des vaincus n'a d'égale que le paternalisme autori-

22 Mandements des évêques de Québec, édit. H. Têtu et C.-O. Gagnon, Québec, Côté, 1888, vol. 2, p. 264. 
taire des vainqueurs ${ }^{23}$. Le discours du vainqueur, comme celui du vaincu, manifestant la plus parfaite duplicité, on imagine mal que le groupe en position d'infériorité ait pu, sans amertume ni déchirement, se livrer à pareil théâtre courtisan. Quant à l'autorité religieuse, les Canadiens ne pouvaient que s'en méfier, quand ils voyaient l'évêque Briand multiplier les mandements par lesquels il se montrait si dévoué à l'autorité britannique et exprimer à l'occasion le plus profond mépris pour ceux qu'il appelait ses «chers Canadiens». Justifiant en juin 1776 l'excommunication dont avaient été frappés ceux qui avaient encouragé le parti des insurgés ou qui s'étaient révoltés contre leurs prêtres, il rappelait les circonstances où l'abbé Bailly de Messein avait été blessé:

circonstances, ajoutait-il, qui feront à jamais la honte de la nation canadienne et qui la dépeindront comme une nation d'une cruauté et d'une barbarie plus que sauvage, et qui ont fait dire aux Bostonnais eux-mêmes indignés, que, s'ils avaient un Canadien à faire rôtir, ils en trouveraient cent autres pour tourner la broche (Mandements des évêques, vol. 2, p. 277-278).

La neutralité des Canadiens en 1775 vient-elle de ce qu'ils ont été gagnés par l'esprit révolutionnaire qui souffle au sud? Les Canadiens sont-ils vraiment tentés par les idées d'émancipation de leurs voisins? Carleton semble le croire quand il affirme que «tout esprit de subordination est détruit» chez eux (DRHCC, 1759-1791, p. 651). Ou bien sont-ils simplement «en proie à une [...] confusion d'ignorance, de crainte, de crédulité, de perversion et de préjugé», comme l'estime William Hey (lettre au Lord Chancelier, 28 août 1775, DRHCC, 1759-1791, p. 656-657)? Peut-être Carleton et Hey ont-ils raison, même si leurs propos fleurent la déception et le mépris. Bousculés par les événements, les Canadiens adoptent, me semble-t-il, une attitude attentiste guidée par une sorte de prudence paysanne qui refuse de s'engager sur un terrain mal connu. Que certains aient été attirés par les idées d'indépendance et de liberté semble probable. A coup sûr, la fondation, en 1778, de la Gazette littéraire,

23 De cela, Briand était bien conscient, qui écrivait dans son mandement de 1776: «Vous avez irrité votre Souverain, à la vérité, mais il est bon [...]. Mais si vous persistez dans votre révolte, vous forcerez aux plus rigoureux sentiments. [...] Quelle a dû être la surprise de l'Angleterre, lorsqu'elle a été informée de votre défection, de votre désobéissance, de votre révolte et de votre réunion à des esprits rebelles! Mais quelle doit être aussi sa colère et son indignation contre vous! N'avez-vous pas lieu de craindre que sa bonté trompée ne se tourne en fureur et qu'elle ne vous accable de châtiments, au lieu des faveurs dont elle vous a comblés jusqu'ici [...]?» (ibid., p. 270-271). 
un journal d'idées sur le modèle français, animé par Valentin Jautard et Fleury Mesplet, qui seront de passionnés défenseurs des Lumières européennes, les initie aux débats d'idées et leur fera prendre conscience du pouvoir de l'écrit sur l'opinion publique. Bien plus, la Gazette littéraire donnera l'occasion à de jeunes intellectuels ou à des étudiants des sulpiciens de faire leurs premières armes.

La décennie qui s'ouvre avec l'instauration de l'Acte constitutionnel en 1791 me semble cruciale pour la consolidation de l'identité canadienne, car les descendants des colons français se familiarisent avec le parlementarisme. Les deux Canadas, placés sous l'autorité d'un gouverneur général, sont dotés d'une assemblée élue par les censitaires, d'un conseil législatif et d'un conseil exécutif nommés par le gouverneur. Pour obtenir la sanction royale de Londres, toute loi votée par les assemblées doit recevoir l'approbation du gouverneur qui détient ainsi un droit de veto. Lors de la première élection au Bas-Canada en 1792, 15 députés de langue anglaise sont élus, contre 35 de langue française. La majorité des membres des conseils exécutif et législatif sont de langue anglaise. Dès les premiers débats parlementaires, Britanniques et Canadiens se divisent sur l'élection d'un président d'assemblée et sur la langue à utiliser pour les débats et les lois. Majoritaires à l'assemblée, les Canadiens se rendront vite compte qu'ils ne détiennent pas beaucoup plus qu'un pouvoir moral de suggestion. Aussi chercheront-ils bientôt à déplacer le débat vers la scène publique, lorsque l'un des leaders, Pierre Bédard fondera en 1806, avec François Blanchet, le journal le Canadien, pour défendre sur la place publique l'intérêt de ses compatriotes et mieux lutter contre l'oligarchie anglaise regroupée autour du gouverneur Craig ${ }^{24}$. On ne sera donc pas surpris que l'autoritaire Craig fitt saisir les presses et enfermer Bédard et Blanchet, que leurs électeurs réélirent quand même. Avec ses faucons du Conseil exécutif (l'archevêque Mountain, le juge loyaliste Sewell, le greffier Ryland), Craig fera voter une sorte de loi des mesures de guerre, craignant, prétendait-il, que le gouvernement fût renversé.

Au moment même où ils apprennent à utiliser la Chambre d'assemblée et qu'ils découvrent le pouvoir des journaux, les Canadiens pren-

24 Le Canadien était aussi fondé pour faire contrepoids à l'organe de la bourgeoisie commerçante de Québec, le Daily Mercury, qui ridiculisait les Canadiens et se moquait de leurs parlementaires. On ne s'étonnera pas que l'évêque Plessis, le 4 décembre 1809 , manifeste à nouveau sa servilité envers le pouvoir anglais, lorsqu'il condamne le Canadien qui inculque à ses lecteurs - même des membres du clergé l'insubordination, voire la révolte ouverte. 
nent connaissance des événements et des idées qui agitent la France depuis le début de la Révolution. Certaines brochures, comme celle de 8 pages du "citoyen Genêt», ambassadeur de France aux États-Unis, toucha un certain nombre de personnes: les Français libres à leurs frères canadiens. Commençant par la célèbre phrase de Rousseau «L'homme est né libre», elle invite les Canadiens à se libérer $\mathrm{d}^{\prime}$ «un gouvernement [...] qui est devenu le plus cruel ennemi de la liberté des peuples»: «Le pays que vous habitez a été conquis par vos pères. [...] Cette terre vous appartient. Elle doit être indépendante ${ }^{25}$.»Certains, comme le procureur général James Monk, semblent saisis de panique, car ils craignent de trouver dans le Bas-Canada «la même barbarie sauvage qui s'est exercée en France». Dans sa lettre du 26 novembre 1793 au secrétaire d'État à l'Intérieur Henry Dundas, il en attribue la responsabilité à des écrits comme ceux de Genêt et annonce qu'il a fait adopter une loi pour «suspendre l'habeas corpus dans les cas de suspicion, de trahison, pour interdire les rassemblements, pour prévenir la venue d'étrangers, pour interdire les discours séditieux et la propagande de fausses nouvelles» (Lacoursière, ibid., p. 35). A la même époque, craignant une attaque de la France, les autorités coloniales promulguent la nouvelle loi de la milice, qui oblige tout résident du Bas-Canada, de 18 à 60 ans, à servir comme milicien. L'application de la loi soulève des manifestations assez vives (Monk parle d'émeutes), dès juin 1794, parce que plusieurs craignent d'être envoyés hors du pays. Plusieurs Canadiens seront emprisonnés au cours de ces années agitées. Avec la victoire de Nelson sur la flotte française à Aboukir, le $1^{\text {er }}$ août 1798 , la crainte d'une attaque française disparaît et le gouverneur Prescott proclame le 10 janvier 1799 jour d'action de grâce où l'on remerciera Dieu «pour l'intervention de sa divine Providence dans cette victoire signalée sur notre ennemi» (Lacoursière, ibid., p. 52). La hiérarchie catholique ne sera pas en reste, puisqu' on chantera le Te Deum dans toutes les églises et que le coadjuteur d'origine canadienne, Plessis, dans son sermon de la cathédrale de Québec, se réjouira de ce "glorieux événement» où Dieu «a terrassé nos ennemis perfides»: «tout ce qui affaiblit la France tend à l'éloigner de nous. Tout ce qui l'en éloigne assure nos vies, notre liberté, notre repos, nos propriétés, notre culte, notre bonheur» (Lacoursière, ibid., p. 59). Son supérieur, l'évêque Denaut, avait manifesté une certaine hésitation à cautionner aussi manifestement le pouvoir civil, parce que la chose ne 
s'était «jamais faite». La Chambre d'assemblée votera de son côté des crédits de 20000 livres pour aider l'Angleterre dans son effort de guerre contre la France.

Je n'ironiserai pas sur cet étrange providentialisme qui unit d'une même voix le prélat catholique et le gouverneur protestant pour célébrer un "glorieux événement», comme le disait Plessis. Je veux plutôt souligner que la démarcation ne s'établit pas d'après la foi religieuse ou l'origine ethnique, mais par rapport au pouvoir politique, comme s'en rendront bien compte ceux qu' on appellera «les Patriotes» de 1837-1838.

Je ne m'aventure pas plus loin dans le $\mathrm{XIX}^{\mathrm{e}}$ siècle, car on voit déjà se dessiner les conflits qui atteindront leur point culminant lors de ces rébellions de 1837-1838, provoquées par le fait que les Canadiens prirent une conscience de plus en plus aiguë que les dés politiques étaient pipés: l'assemblée des élus du peuple ne pouvait jouer son rôle face à l'exécutif nommé par Londres. La révolte fut mâtée et l'Acte d'Union des deux Canadas, qui visait à minoriser les Canadiens, tout en leur faisant assumer les dettes du Haut-Canada, fut votée en 1840. Le gouvernement responsable viendra en 1848 et le clergé établira graduellement son pouvoir dans les domaines de l'éducation et des services sociaux, ce qui permettra aux Canadiens français de compenser ainsi leur impuissance sur le plan politique. Mais la date la plus importante sans doute, dans la perspective de l'identité canadienne puis canadienne-française, est 1867: par l'Acte de l'Amérique du Nord britannique, qui réunit le NouveauBrunswick, la Nouvelle-Écosse, l'Ontario et le Québec, la survie et l'identité des Canadiens français paraissent assurées, puisque, dans la province de Québec où ils sont largement majoritaires, ils auront un gouvernement provincial à eux. Ils peuvent même espérer se développer au Manitoba, un an après la rébellion des Métis: devenant la cinquième province du Canada, le Manitoba garantit en effet aux Canadiens des écoles séparées et reconnaît l'anglais et le français comme langues officielles. La suite est connue. En l'espace de dix ans, de 1870 à 1880, avec les nombreux immigrés venus d'Europe et surtout la migration de Britanniques ontariens, le Manitoba devient majoritairement anglais. Le Canada hors Québec ne sera jamais binational. Les communautés canadiennes-françaises survivront difficilement au milieu de combats sans fin pour conserver leurs écoles et leurs institutions.

\section{Conclusion}

Même si l'entreprise est périlleuse, tentons néanmoins de synthétiser quelques traits de cette identité canadienne telle qu'elle a évolué sous les Régimes français et anglais. Je n'insiste pas sur la langue française et la 
religion catholique, constitutives du noyau dur de l'identité. Je parlerai plutôt du couple nomadisme/sédentarité qui me paraît marquer dès l'origine cette identité. D'une part, la rareté de la main-d'œuvre, l'abondance des terres et la dispersion de la population permettent d'échapper jusqu'à un certain point à la contrainte du pouvoir politique et religieux; d'autre part, une partie de la population masculine nomadise avec les Amérindiens dont elle partage l'existence plus ou moins continûment. Certains voyageurs des pays d'En-Haut s'y établiront pour donner naissance à une population métisse qui augmentera rapidement au XIX ${ }^{e}$ siècle et dont on connaît l'histoire tragique. Cette part nomade que porte en elle la société canadienne se manifeste dans tout un ensemble de références et de pratiques quotidiennes égalitaristes que les administrateurs nomment «indépendance d'esprit, insolence, fierté, insoumission ou ensauvagement». Mais, en même temps qu'ils déplorent cette distanciation culturelle, les administrateurs se rendent compte que cette indépendance, cette fierté ombrageuse s'accompagnent de qualités guerrières remarquables pour assurer la sauvegarde de la colonie. Tous ces traits construisent, dès la première génération née au Canada, une nouvelle identité qui se pose différente de l'identité française. Bien sûr, elle ne s'exprime pas collectivement de manière cohérente dans une démarche politique concertée ou dans la publication de journaux, puisque la situation coloniale ne le permet pas.

Avec le Régime anglais commence une nouvelle phase de l'identité, marquée d'abord par le choc de la défaite militaire. Que certains aient pu tirer profit du nouveau régime ne change rien au fait qu'il y a eu rupture, que le changement de métropole a empêché la consolidation d'une bourgeoisie canadienne au moment où les pays européens étaient travaillés par la montée de la bourgeoisie qui faisait émerger de nouvelles valeurs dominantes.

Sur un autre plan, celui du rapport au territoire, la situation a dramatiquement changé. Avec l'amputation du Labrador et des régions des Grands Lacs et de l'Ohio, les habitants de la nouvelle Province of Quebec perdent leur espace de nomadisme et, à toutes fins pratiques, leurs alliances étroites avec les Amérindiens. La Conquête les force à la neutralité face aux révoltes amérindiennes contre l'Angleterre comme celle de Pontiac, ancien allié des Français. Comme les Indiens deviennent les alliés et les sujets des Anglais, le lien politique qui subsiste entre Canadiens et Indiens passe désormais par le maître anglais. Le pôle terrien des Canadiens se trouve renforcé au détriment de leur pôle nomade. Si cette sédentarité plus grande peut développer des solidarités horizontales ou même une forme de civilité que vantent les observateurs de passage, elle établit une coupure radicale dans la verticalité du politique. La tendance égalitariste que plusieurs ont notée tente de s'accommoder 
d'une organisation politique où les élites aristocratique et religieuse canadiennes deviennent les vassaux des vainqueurs de 1763, conformément à la logique de l'indirect rule britannique. A l'extérieur de cette Province de Québec, vers le sud et vers l'ouest, les voyageurs canadiens continueront de servir d'intermédiaires entre les Amérindiens et les marchands, désormais britanniques (le plus souvent écossais). En plus d'être pourvoyeurs de fourrures, ils deviendront guides d'explorateurs comme Mackensie (Laurent Leroux), qui atteint la mer de Beaufort en 1787, ou encore les Américains Lewis et Clark (Charbonneau et Tabeau), vers l'ouest américain en 1804 . Avec le blocus de Napoléon, ils deviendront forestiers pour les marchands de bois anglais ${ }^{26}$.

Tout en balisant leur nomadisme dans le cadre de l'empire britannique et en réduisant leur espace d'indépendance, le Régime anglais permettra aux Canadiens d'investir graduellement le politique et de s'affirmer dans la sphère publique par les journaux. Leurs premières pétitions aux administrateurs londoniens leur donneront conscience d'un pouvoir d'intervention qu'ils utiliseront quelques décennies plus tard quand ils deviendront membres d'une Chambre d'assemblée et fonderont des journaux pour agir dans l'espace public. Leur rhétorique du sujet respectueux, reconnaissant et soumis se teintera de plus en plus d'une thématique où le droit et la liberté civile auront une large place. Tout en restreignant leur espace territorial, la constitution de 1791, si limitée soit-elle, ouvre donc la porte à une prise en mains du politique. Et c'est justement cette volonté de prise en mains qui se manifestera, au début du XIX ${ }^{e}$ siècle, pour aboutir aux rébellions de 1837-1838: la majorité parlementaire du Bas-Canada, comme celle du Haut, n'accepte plus que l'exécutif qui gouverne soit choisi en dehors d'elle. Dans le Bas-Canada, la crise se doublera d'une tension ethnique, puisque le pouvoir exécutif était entre les mains de protestants britanniques qui dominaient l'économie. Cette crise débouchera en 1840 sur l'Acte d'Union des deux Canadas qui mettra les Canadiens de langue française en minorité, comme le craignaient les «Patriotes» de 1837. Avec la montée du pouvoir religieux, à partir des années 1850, les Canadiens compensent leur impuissance politique par divers modes d'affirmation collective fondés sur les paroisses catholiques qui ont leurs écoles et leurs embryons de services sociaux. D'inévitables conflits ont éclaté qui ont entraîné des conséquences très graves pour la survie canadienne-française aux États-

26 Beaucoup plus tard, en 1874, on verra même Prudent Beaudry devenir maire de Los Angeles, pendant que son frère Jean-Louis l'est de Montréal. 
Unis et, dans une moindre mesure, au Canada anglais. A ces conflits entre des communautés francophones catholiques et un gouvernement de langue anglaise se sont ajoutés l'urbanisation, la scolarisation et le milieu de travail en anglais qui ont joué dans le même sens.

La Confédération de 1867 redonnera partiellement aux vaincus de 1760 le pouvoir politique sur un espace délimité où ils seront majoritaires. Mais cette consolidation du pouvoir politique au Québec se produira en même temps qu'une extinction graduelle des communautés franco-américaines et une assimilation plus ou moins rapide des Canadiens français hors Québec. De cette anglicisation des leurs, les habitants francophones du Québec ont tiré la conviction que seule une assise politique solide garantirait leur survie. Et ils l'ont affirmé dans leur manière de se désigner eux-mêmes. Après s'être appelés Canadiens, Canadiens français, ils se sont mis, après 1960, à se dénommer Québécois, pour se situer face aux autres Canadiens français. Il ne s'agit pas là d'un repli frileux sur une «ethnicité tribale» désuète, comme se plaisent à le répéter Mordecai Richler, Diane Francis et quantité d'autres ${ }^{27}$. Plutôt qu'un refermement «tribaliste», le recentrement politique sur le Québec trahit la conscience de la fragilité d'une identité minoritaire au Canada,

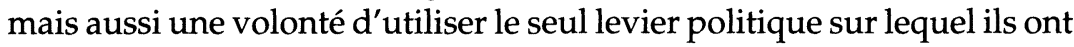
vraiment prise. Les Québécois sont aussi sensibles à la diminution du

27 Jour après jour, les Québécois sont profondément choqués quand, dans les médias anglophones, des caricatures, des lettres de lecteurs ou des columnistes leur accolent des qualificatifs qui les assimilent aux nazis ou au fascistes, ou encore parlent de «nettoyage ethnique». Je ne rappellerai pas la crise d'Oka, sur laquelle on peut lire le beau livre de Réal Brisson: Oka par la caricature. Deux visions distinctes d'une même crise, Sillery (Québec), Septentrion, 2000. Je mentionnerai seulement, parmi tant d'autres, l'article de Daniel Sanger paru dans Saturday Night de mars 2000: «Colder and whiter. In Vieux Québec, ethnic cleansing occurs by attrition.» Si D. Sanger avait simplement ouvert un annuaire téléphonique et essayé de comprendre les migrations de populations depuis 1970, il aurait vu que la petite communauté chinoise de Québec, par exemple, n'a pas subi de nettoyage ethnique: elle a fait comme les Québécois de vieille souche du même quartier qui ont déménagé à Montréal, ou encore se sont installés en banlieue, tout comme l'Université Laval qui a émigré à Sainte-Foy, bien loin du Quartier latin. Tout comme mes quatre enfants qui gagnent maintenant leur vie à Montréal. Quant à Diane Francis, elle écrivait, dans le National Post du 10 avril 2000: "This is brown shirts a la Quebec who hope to fan fears about the future of the pur laine Super Race.» Imagine-t-on le tollé indigné que provoquerait, à Toronto, Halifax ou Vancouver, l'utilisation d'un vocabulaire aussi offensant par un journaliste d'Actualité ou des Affaires pour qualifier la population anglophone de l'Ontario ou des Maritimes? Que sont devenus ces fiers champions de la morale civique, si âpres à dénoncer la malheureuse déclaration postréférendaire de Jacques Parizeau (qui, du reste, démissionna immédiatement après)? 
pouvoir politique à Québec que le reste du Canada peut l'être à l'indépendance du Québec. Aussi longtemps qu'on ne transcrira pas cette position en politique concrète, le Québec et le reste du Canada continueront à se regarder comme des chiens de faïence et consommeront un peu plus ce que Christian Dufour appelle la Rupture tranquille (Montréal, Boréal, 1992).

Et, comme si la situation n'était pas encore assez compliquée, il faut y ajouter encore que l'identité québécoise est marquée par une tension continuelle entre Montréal et le reste du Québec. Cette tension me paraît venir de deux facteurs principaux: d'une part, Montréal est un pôle économique et culturel très fort; $d$ 'autre part, contrairement au reste de la province, Montréal est une grande ville multiethnique qui a conservé des institutions anglophones prestigieuses: hôpitaux, universités, etc. Les tensions interethniques ne me paraissent pas venir d'abord de la cohabitation quotidienne, car on remarque des signes évidents $d$ 'intégration harmonieuse, dans les domaines du divertissement, des arts (la littérature, la musique, le théâtre, notamment) et de la recherche scientifique $^{28}$. En revanche, quand il s'agit du projet politique, les néoQuébécois forment avec la communauté anglophone un bloc compact contre le projet d'indépendance qui rassemble $60 \%$ des Québécois de vieille souche francophone.

Puisque je suis déjà sur un terrain glissant, je m'avancerai encore un peu plus loin pour comparer brièvement ce que j'appellerai, faute d'un meilleur terme, les réalités identitaires canadienne anglophone et québécoise. Pour moi, cette réalité a beaucoup à voir avec la Conquête de 1760 . Et cette Conquête, quoi qu'on dise, n'est pas une simple rencontre entre Britanniques, Français et Amérindiens. Elle est d'abord haine réciproque, violence, victoire armée des uns sur les autres. Par opposition à l'identité canadienne puis québécoise dont j'ai longtemps parlé, l'identité canadienne-anglaise s'est édifiée plus récemment et de manière différente. Elle s'est construite d'abord sur une immigration loyaliste lors du grand maelström des années 1775 , alors que quelques dizaines de milliers de personnes, voulant demeurer Britanniques, ont refusé de devenir «Américains» et ont estimé que les Canadiens papistes et français devaient être traités comme des vaincus. Après se sont ajoutés des immigrants des îles britanniques, surtout d'Irlande. Entre 1817 et 1834, par exemple, la population irlandaise du Canada passa de 5000 à

28 Nommons seulement pêle-mêle Brathwaite, Kokis, Kavannah, Laferrière et pensons, chaque année aux prix du Québec et de l'Association canadienne-française pour l'avancement des sciences. 
52 000; en 1847 seulement, 50000 Irlandais catholiques passèrent au Canada. Formant des communautés catholiques nombreuses et compactes, ces groupes se trouvèrent vite en conflit avec les Canadiens français dont ils partageaient parfois les mêmes écoles et les mêmes églises. On imagine facilement aussi les tensions qui naquirent entre les loyalistes britanniques et ces immigrants irlandais qui avaient de bonnes raisons d'en vouloir à l'Angleterre. A ces deux groupes serrés qui construisent une première canadianité anglophone s'ajouteront ces vagues successives venues d'Europe centrale, d'Italie ou d'ailleurs qui ne veulent rien savoir des vieilles querelles britannico-françaises. Pour eux, les Canadiens de vieille souche française ne sont qu'un groupe parmi d'autres, comme l'affirmera d'ailleurs plus tard la politique fédérale du multiculturalisme. L'image de la "mosaïque canadienne» leur semblera beaucoup plus adéquate que celle des "deux peuples fondateurs», surtout qu'il faut y joindre les Amérindiens, avec leurs revendications territoriales et politiques. Cette attitude, si naturelle soit-elle, heurte la vieille communauté francophone pour qui une identité collective et une culture ne sont pas des pièces de mécano qu'on bricole comme on veut; elles se construisent dans le temps, génération après génération, et se transforment en agrégeant des éléments nouveaux. Dans cette optique, il faut bien admettre que l'identité québécoise, si elle partage plusieurs traits avec l'identité canadienne, s'en distingue aussi radicalement et n'entretient pas le même rapport au politique.

On comprend donc que la seule porte de sortie pour tout le monde serait d'en arriver à ce qu'on a appelé une fédération canadienne asymétrique où les pouvoirs du gouvernement du Québec ne seraient pas exactement les mêmes que ceux des autres provinces ou régions du pays. Mais le vent ne paraît pas souffler de ce côté. L'échec de l'accord du lac Meech et le coup de force constitutionnel de 1982, qui laisse aux Québécois l'amertume de s'être fait flouer, ne demeure qu'un mauvais souvenir à oublier pour le reste du Canada.

RÉAL OUELLET

Université Laval 